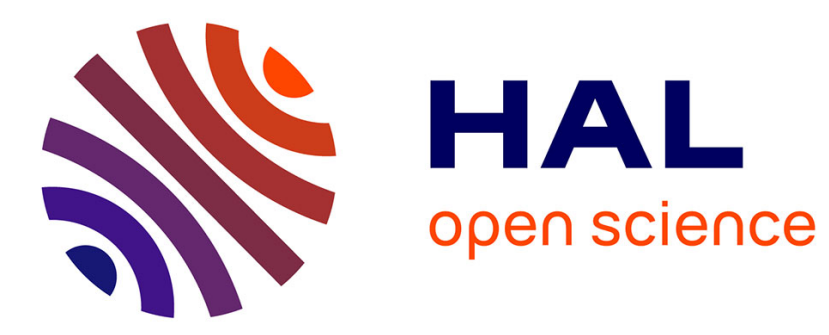

\title{
MODÉLISATION DE L'INTERACTION LATÉRALE D'UNE ONDE DE DÉTONATION AVEC LE BERYLLIUM. VALIDATION EXPÉRIMENTALE ET NUMÉRIQUE
}

J. Servas, J. Aveille, N. Carion

\section{To cite this version:}

J. Servas, J. Aveille, N. Carion. MODÉLISATION DE L'INTERACTION LATÉRALE D'UNE ONDE DE DÉTONATION AVEC LE BERYLLIUM. VALIDATION EXPÉRIMENTALE ET NUMÉRIQUE. Journal de Physique IV Proceedings, 1991, 01 (C3), pp.C3-803-C3-811. 10.1051/jp4:19913113 . jpa00249916

\section{HAL Id: jpa-00249916 https://hal.science/jpa-00249916}

Submitted on 1 Jan 1991

HAL is a multi-disciplinary open access archive for the deposit and dissemination of scientific research documents, whether they are published or not. The documents may come from teaching and research institutions in France or abroad, or from public or private research centers.
L'archive ouverte pluridisciplinaire HAL, est destinée au dépôt et à la diffusion de documents scientifiques de niveau recherche, publiés ou non, émanant des établissements d'enseignement et de recherche français ou étrangers, des laboratoires publics ou privés. 
Colloque C3, suppl. au Journal de Physique III, Vol. 1, octobre 1991

\title{
MODÉLISATION DE L'INTERACTION LATÉRALE D'UNE ONDE DE DÉTONATION AVEC LE BERYLLIUM. VALIDATION EXPÉRIMENTALE ET NUMÉRIQUE
}

\author{
J.M. SERVAS, J. AVEILLE et N. CARION \\ Commissariat à I'Énergie Atomique, Centre d'Études de \\ Vaujours-Moronvilliers, BP. 7, F-77181 Courtry, France
}

RESUME. Nous présentons les résultats expérimentaux obtenus lors de l'interaction irrégulière d'une onde de détonation avec une plaque de béryllium. Un modèle décrivant la phénoménologie de cette famille d'interaction est proposé et une simulation numérique valide ce modèle.

Abstract - This paper presents the experimental results of the irregular interaction of an oblique detonation wave with a beryllium sample. Two heterogeneous high explosives are used (TATB and HMX based compositions). Then, these results are interpreted by a model founded on an analogy between a supersonic flow past a wedge and the refraction at the explosive/beryllium interface. A numerical simulation improves our knowledges on these flows and validate the proposed model.

\section{INTRODUCTION}

La réfraction d'une onde de détonation oblique à l'interface avec un milieu connexe a fait l'objet, au cours des vingt dernières années, de nombreuses publications. L'intérêt porté à ce type d'étude provient de l'absence de solution unique englobant toutes les configurations possibles. Celles-ci se subdivisent schématiquement en trois groupes :

- le premier correspond à la réfraction régulière pour laquelle une solution simple est immédiate,

- le second groupe est constitué par les écoulements avec onde de Mach dans les produits de détonation. Le modèle proposé en 1965 par STERNBERG et PIACESI /1/ rentre dans le cadre de cette interaction qui survient pour le couple pentolite/fer pour un angle d'incidence de la détonation compris entre 63 et $77^{\circ}$.

- le troisième groupe comprend les couples pour lesquels le matériau inerte a une vitesse du son plastique élevée par rapport à la vitesse de détonation de l'explosif. EDEN et WRIGHT ont ainsi mis en évidence /2/ un choc précurseur dans l'aluminium lorsqu'il est associé au baratol. Récemment, EDEN et BELCHER /3/ ont effectué des expériences sur un couple ayant un comportement similaire : 
Ec35/béryllium. De son côté, T.NEAL /4/ avait étudié le couple composition B3/béryllium.

La présente étude est consacrée à deux couples explosif/inerte présentant tous deux une interaction irrégulière :

- le premier est constitué d'un explosif au TATB (appelé par la suite T2) associé à deux plaques de béryllium. Pour ce couple, la vitesse de détonation de l'explosif est inférieure à la vitesse du son plastique du matériau connexe.

- le second couple est constitué d'un explosif à l'octogène (appelé par la suite X1) également associé à deux plaques de béryliium. Pour cette configuration, la vitesse de détonation de l'explosif est supérieure à la vitesse du son plastique du matériau connexe.

Dans un premier temps, nous décrirons le montage ainsi que les diagnostics permettant d'avoir accès aux caractéristiques principales de ce type d'interaction puis, nous proposerons un modèle analogique expliquant la phénoménologie des écoulements observés. Enfin, ce modèle sera confirmé par une étude numérique.

\section{ETUDE EXPERIMENTALE}

\subsection{Description du montage et des diagnostics associés.}

Le schéma de principe du montage est présenté sur la figure 1, cidessous :

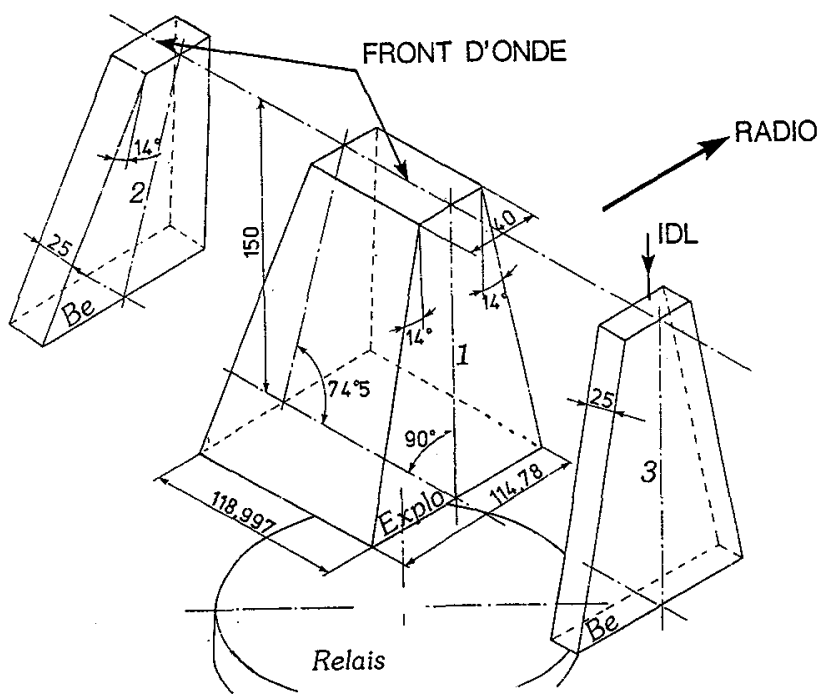

Figure 1 - schéma de principe du montage

Il est constitué :

1) D'un système d'amorçage.

2) D'un bloc prismatique d'explosif X1 ou T2. Ce bloc est usiné aux angles $\eta=74,5^{\circ}$ et $90^{\circ}$ pour deux faces et à $14^{\circ}$ pour les deux autres faces.

3) De plaques de béryllium d'épaisseur e $=25 \mathrm{~mm}$ disposées sans plan de collage de part et d'autre de l'explosif. 
Quelques caractéristiques des explosifs X1 et T2 sont reportées dans le tableau 1.

Tableau 1. Caractêristiques des explosifs X1 et T2

\begin{tabular}{|c|c|c|c|c|}
\hline composition & $\begin{array}{c}\rho \mathrm{o} \\
\mathrm{g} / \mathrm{cm}^{3}\end{array}$ & $\begin{array}{l}\mathrm{D}_{\mathrm{CJ}} \\
\mathrm{m} / \mathrm{s}\end{array}$ & $\begin{array}{l}\mathrm{P}_{\mathrm{CJ}} \\
\mathrm{GPa}\end{array}$ & $\gamma$ \\
\hline $\mathrm{X} 1$ & 1,822 & 8778 & 34,2 & 3,10 \\
\hline $\mathrm{T} 2$ & 1,855 & 7700 & 29,0 & 2,79 \\
\hline
\end{tabular}

Les coefficients élastoplastiques du béryllium figurent dans le tableau 2 ci-dessous :

Tableau 2. Coeffj rients élastoplastiques du béryllium

\begin{tabular}{|c|c|c|c|}
\hline $\begin{array}{c}\rho \circ \\
\mathrm{g} / \mathrm{cm}^{3}\end{array}$ & $\begin{array}{c}\mathrm{C}_{\mathrm{L}} \\
\mathrm{m} / \mathrm{s}\end{array}$ & $\begin{array}{c}\mathrm{U}=\mathrm{A}+\mathrm{Bu} \\
\mathrm{m} / \mathrm{s}\end{array}$ & $\begin{array}{c}\mathrm{Y} \\
\mathrm{GPa}\end{array}$ \\
\hline 1,845 & 13500 & $\mathrm{U}=8037+1,053 \mathrm{u}$ & 0,3 \\
\hline
\end{tabular}

$C_{L}$ est la vitesse des ondes élastiques longitudinales du béryllium,

U est la vitesse de choc du Be donnée sous la forme $U=A+B u$,

$\mathbf{Y}$ est la limite élastique en traction.

Afin de détecter toutes les ondes (tant élastiques que plastiques) émergeant du dispositif expérimental, nous avons disposé sur la face de sortie un prisme à réflexion totale permettant d'enregistrer au moyen d'une caméra à fente (CF6) la déflexion de la lumière d'un flash à argon lors de l'arrivée des perturbations sur la base du prisme. La totalité de l'explosif et la sortie du béryllium côté incidence $74^{\circ} 5$ sont observés à l'aide de ce diagnostic.

Les ondes de choc et de détonation présentes à l'intérieur du montage avant émergence sont analysées à l'aide d'un diagnostic radiographique.

Un diagnostic I.D.L. a été mis en place pour caractériser le prêcurseur élastique en sortie de la plaque de béryllium côté incidence $\eta=90^{\circ}$.

\section{2 - Résultats obtenus}

Les clichés radiographiques obtenus avec les deux compositions explosives sont comparés sur la figure 2 .

Avec la composition T2 aucune discontinuité de densité ne peut être mise en évidence dans les plaques de béryllium ce qui semble logique puisque, pour une incidence de la détonation $\eta>71^{\circ}$, la vitesse de déplacement de la détonation le long de l'interface est inférieure à la vitesse des ondes plastiques dans le béryllium. 
Le chargement de celui-ci s'effectue donc par un train d'ondes de compression et non par onde de choc.

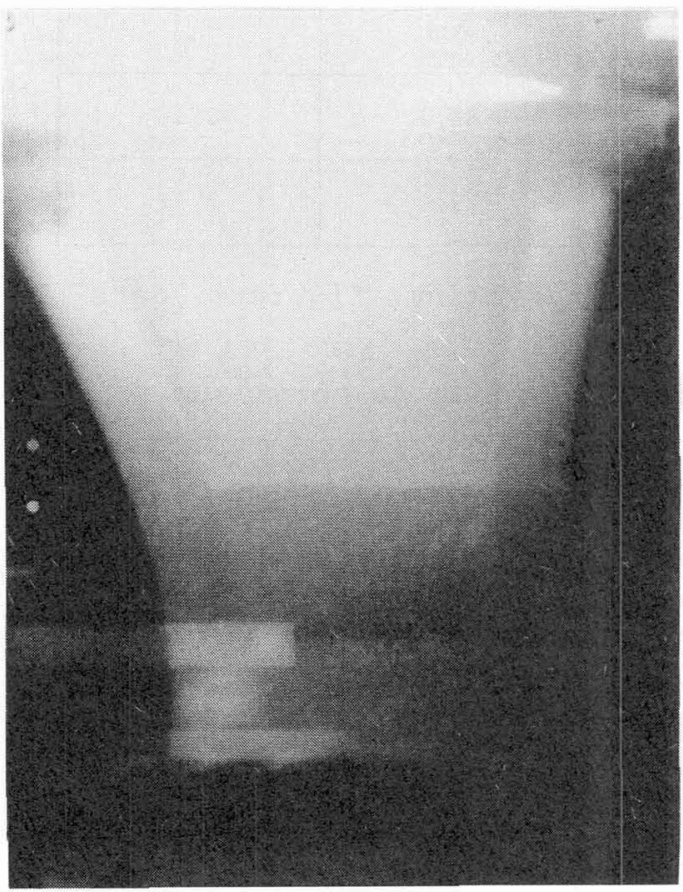

Couple X1/Be

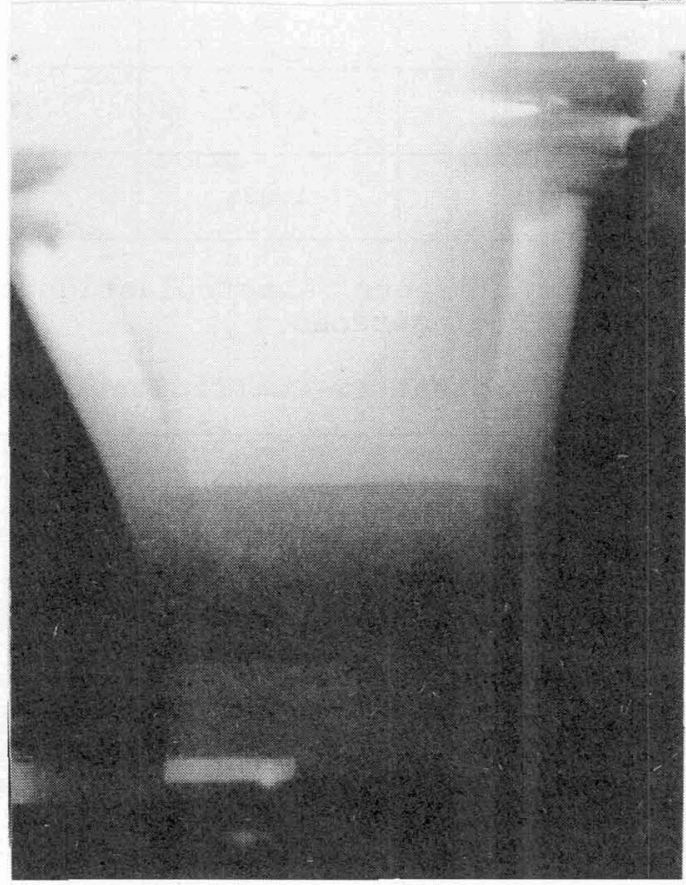

Couple T2/Be

Figure 2 - Clichés radiographiques visualisant les ondes au sein du montage

Pour le couple $\mathrm{X} 1 / \mathrm{Be}$, une onde de choc courbe est nettement visible au sein des deux massifs de béryllium et se propage sensiblement en avant du front de détonation.

Dans le massif explosif, on remarque une avance du front de détonation au voisinage de l'interface $\mathrm{T} 2 / \mathrm{Be}$ pour l'incidence $\eta=74,5^{\circ}$. Par contre, cette avance $n^{\prime}$ existe plus près de l'interface correspondant à $l^{\prime}$ incidence $\eta=90^{\circ}$. En ce qui concerne $I^{\prime}$ interaction $\mathrm{X} 1 / \mathrm{Be}$, l'avance de la détonation est présente au voisinage des deux interfaces.

Le diagnostic optique confirme les observations radiographiques (figure 3) et la sensibilité de la mesure permet de mettre en évidence l'arrivée du précurseur élastique. 


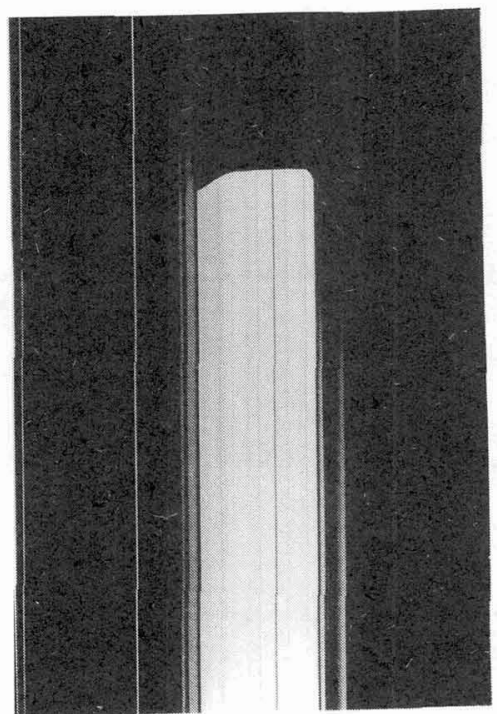

couple $\mathrm{x} 1 / \mathrm{Be}$

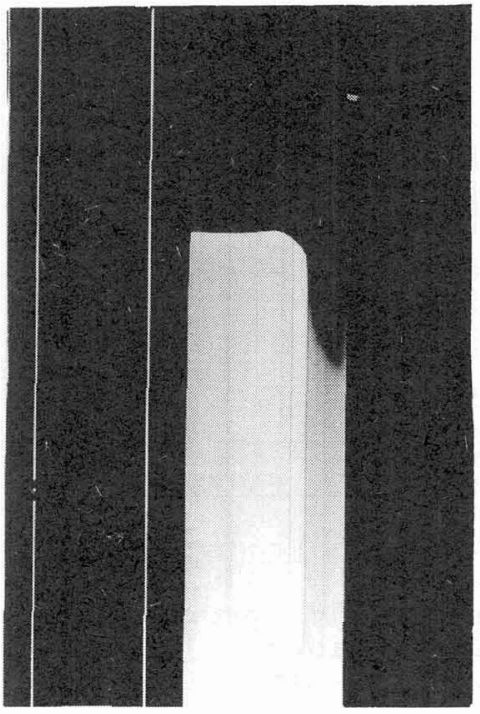

Couple T2/Be

\section{Figure 3 - Enregistrement caméra CF6}

En ce qui concerne le couple $\mathrm{T} 2 / \mathrm{Be}$, on observe une compression de l'explosif par le béryllium qui trouvera une justification dans le chapitre suivant.

Le diagnostic I.D.L. permet d'enregistrer la vitesse de surface libre du béryllium côté $90^{\circ}$. Le signal obtenu, présenté en figure 4 , donne accès aux caractéristiques du précurseur élastique déduites de ce diagnostic et qui sont à comparer aux grandeurs statiques données dans le tableau 2 .

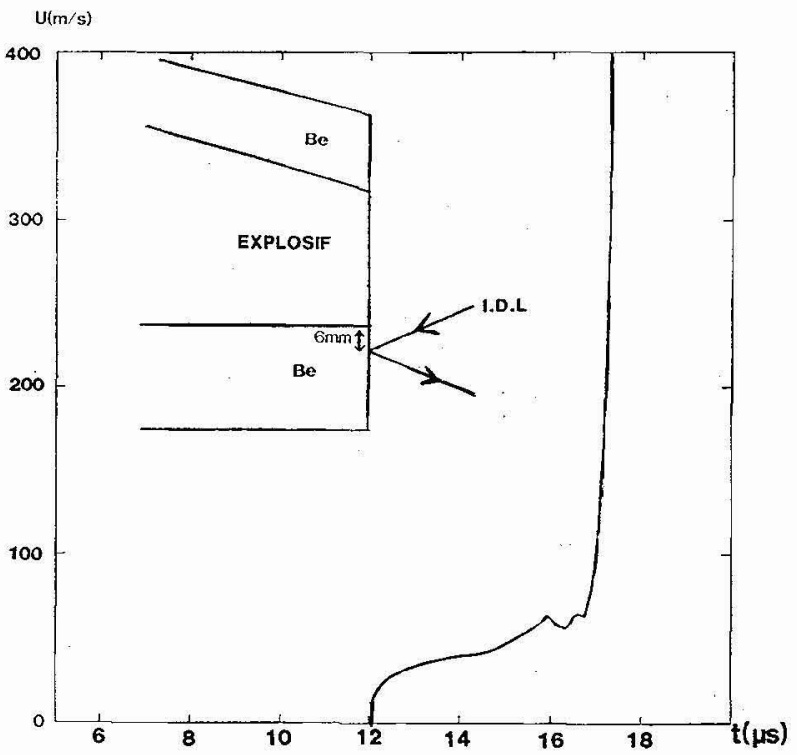

Figure 4 - Mise en évidence expérimentale du précurseur élastique dans le béryllium 
* u élastique $\cong 40-45 \mathrm{~m} / \mathrm{s}$

* $\mathrm{C}_{\mathrm{L}}=13840 \pm 275 \mathrm{~m} / \mathrm{s}$

De ces grandeurs, on déduit la limite élastique d'Hugoniot:

$$
\sigma_{\mathrm{LEH}}=\rho_{\mathrm{o}} C_{\mathrm{L}} \frac{\mathrm{u}_{\text {élastique }}}{2} \cong 0,54 \mathrm{GPa}
$$

\section{INTERPRETATION DES RESULTATS EXPERIMENTAUX PAR UN MODELE DE FLUIDE PARFAIT}

\section{1 - Fondements du modèle}

Le modèle proposé repose sur une analogie entre l'écoulement supersonique d'un fluide autour d'un dière et l'interaction explosif-matériau connexe.

En effet, lorsque l'angle du dièdre est supérieur à $\varphi_{1 \text { lim }}$ (voir figure 5), il n'y a plus de point d'intersection entre la polaire de choc du fluide et la droite $\varphi=\varphi_{1}$. Dans ce cas, 1 'onde de choc se détache pour se stabiliser à une distance finie de la pointe du dièdre.
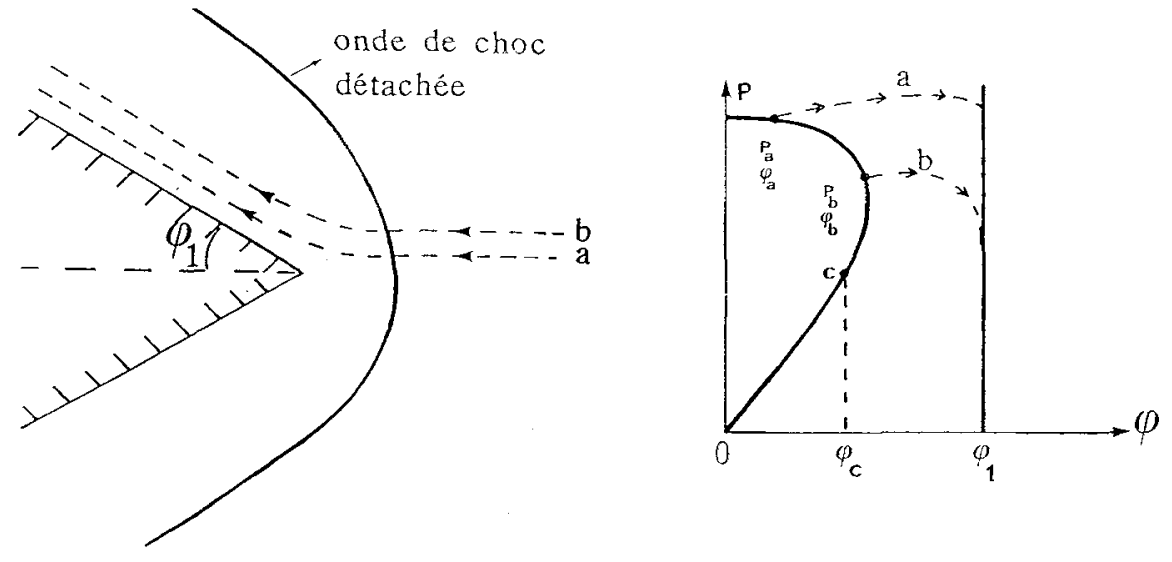

Figure 5 - schéma de principe de l'onde de choc détachêe

Le long de la ligne de courant "a", la pression à l'aval du choc vaut $P_{a}$ et la déflexion $\varphi_{a}$. Ensuite prend place une compression suivie d'une détente isentropique avec augmentation de l'angle de déflexion jusqu'à une valeur proche de $\varphi_{1}$.

\section{2 - Interaction irrégulière X1/Be et $\mathrm{T} 2 / \mathrm{Be}$}

La transposition de cet écoulement à l'interaction XI/Be nous amène au schéma de l'écoulement tel que présenté sur la figure 6 . 

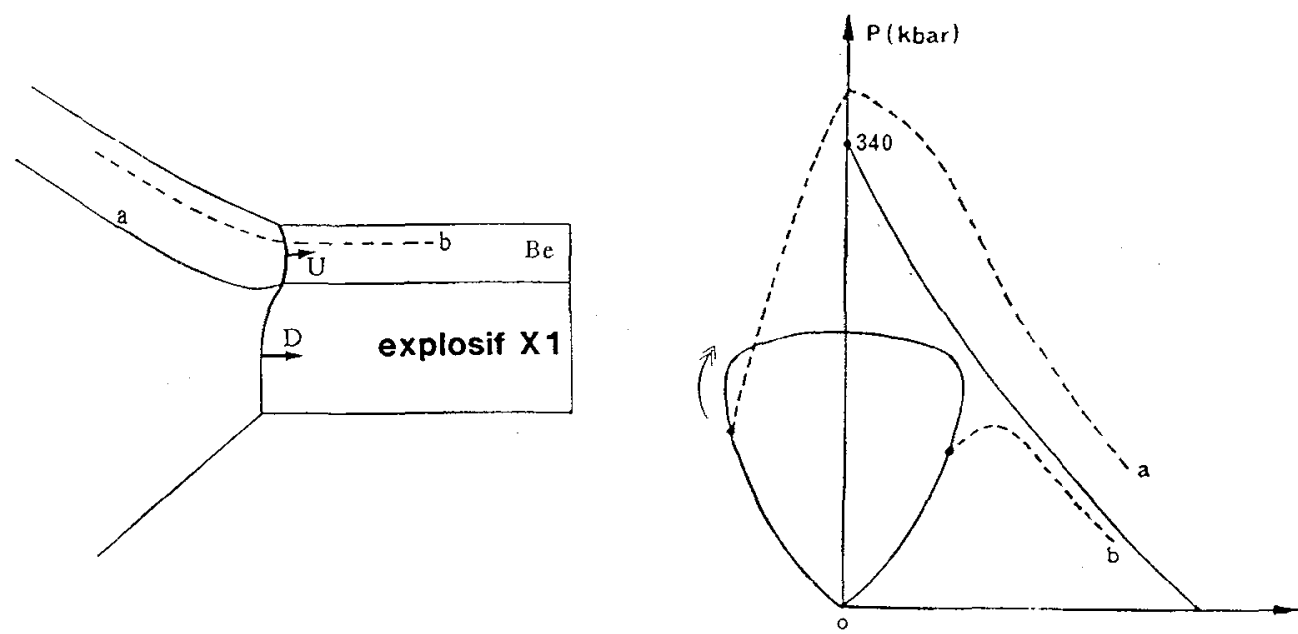

Figure 6 - Schéma de principe de l'interaction explosif $\mathrm{x} 1$ /béryllium

Nous sommes ainsi en présence d'une onde de détonation droite avec une avance de la détonation lorsqu'on approche de l'interface explosif/béryllium. Cette avance est due à la pression exercée par l'onde de choc détachée dans le béryllium (de l'ordre de $11 \mathrm{GPa}$ ) et qui est susceptible de modifier le comportement réactif de l'explosif. Une onde de choc courbe avec un êcoulement aval subsonique est transmise dans le béryllium. Derrière le choc prend place une compression suivie d'une détente isentropique.

Dans le plan pression/angle de déviation, un point situé à l'interface (chemin "a") doit avoir, à l'aval du choc, un angle de déflexion négatif, puis une compression isentropique doit prendre place, suivie d'une détente. Si l'on se rapproche de la surface libre, les conditions à l'aval du choc sont données par les points parcourant la polaire de choc du Be dans le sens de la flèche : la déflexion initiale évolue alors des valeurs négatives vers les valeurs positives (chemin du type "b").

En ce qui concerne l'interaction $\mathrm{T} 2 / \mathrm{Be}$, les caractéristiques de l'écoulement sont sensiblement les mêmes que pour l'interaction $\mathrm{X} 1 / \mathrm{Be}$ à part la polaire de choc du Be qui se réduit au point origine 0 (puisque la vitesse des ondes plastiques est supérieure à la vitesse de détonation) ce qui entraine une absence d'onde de choc et une mise en pression uniquement par ondes de compression.

\section{3 - Comparaison modèle/expérience}

Le modèle précédemment décrit permet d'expliquer la forme de l'onde de choc observée expérimentalement dans le $\mathrm{Be}$. De même les résultats expérimentaux montrant l'avance du front de détonation près de l'interface ainsi que la compression de l'explosif T2 sont cohérents avec le modèle d'onde de choc détachée.

\section{VALIDATION NUMERIQUE DU MODELE}

Une étude numérique a été réalisée à l'aide d'un code bidimensionnel lagrangien. L'avance de la détonation a été simulée à l'aide d'une cinétique de décomposition de type TCD /5/. La figure 7 compare le réseau des isobares obtenu pour les couples X1/Be et $\mathrm{T} 2 / \mathrm{Be}$. 

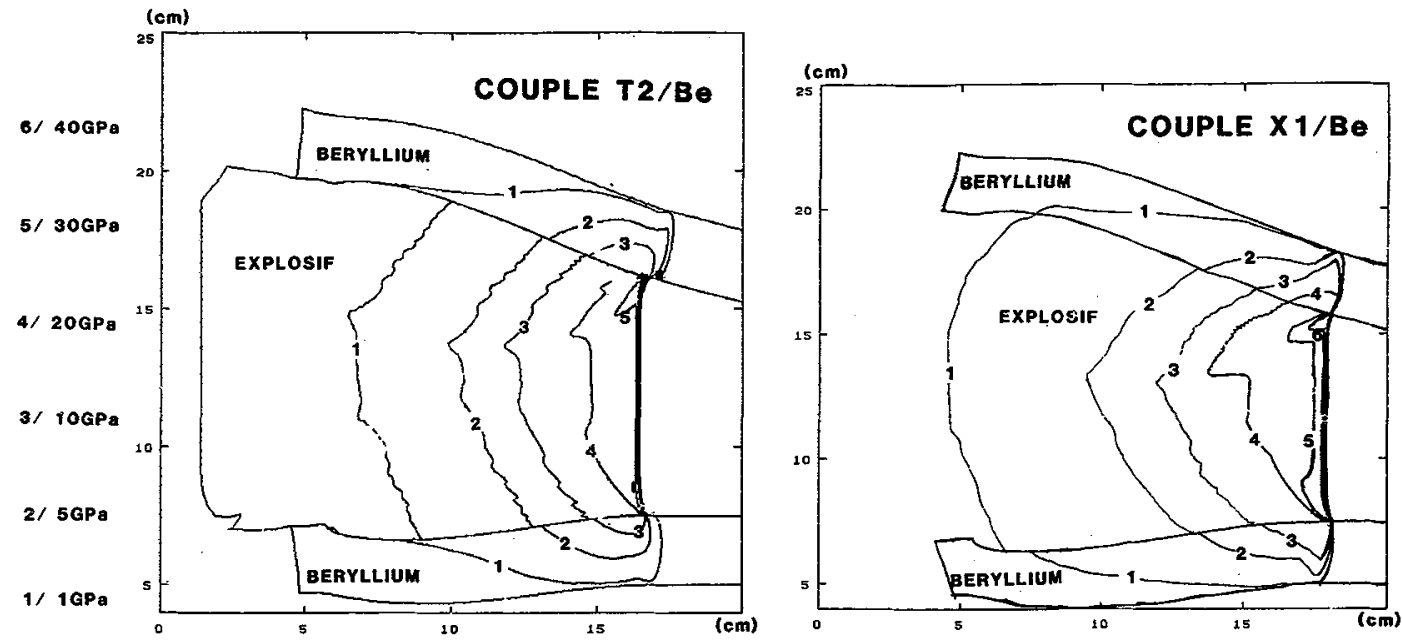

\section{Figure 7 - Réseau d'isobares pour les couples X1/Be et T2/Be}

Les résultats obtenus mettent également en évidence la courbure du choc dans le Be pour le couple X1/Be ainsi que l'avance du front de détonation. Pour le couple T2/Be, la montée en pression dans le béryllium est beaucoup plus étalée, ce qui suggère une absence d'onde de choc.

Les chemins thermodynamiques suivis par différentes particules de Be dans le plan pression/angle de déviation (figure 8) pour le couple $\mathrm{X} 1 / \mathrm{Be}$ mettent en évidence :

1) une pression bien supérieure à la pression maximum admissible par choc,

2) une déflexion initiale négative de l'interface suggèrant une compression de l'explosif.
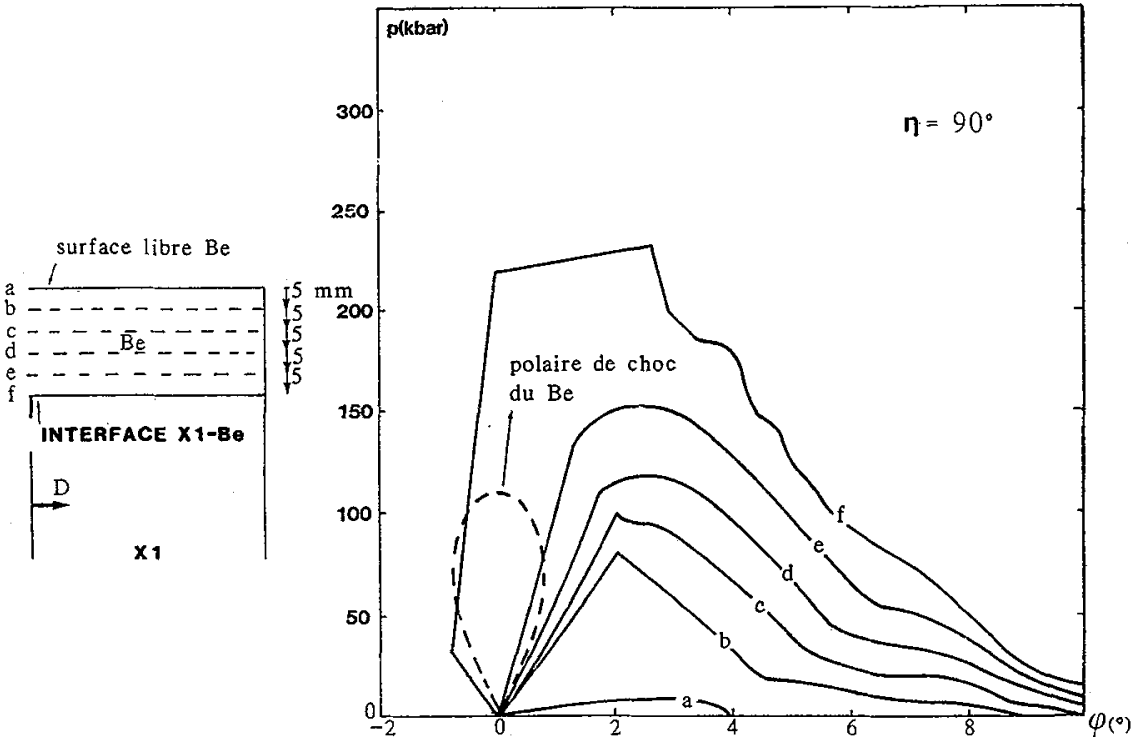

Figure 8 - Chemin thermodynamique dans le plan $(p, \varphi)$ 


\section{CONCLUSION}

Cette étude de l'interaction irrégulière $\mathrm{X} 1 / \mathrm{Be}$ et $\mathrm{T} 2 / \mathrm{Be}$ nous a permis de proposer une interprétation physique originale de cette classe particulière d'écoulements.

Les résultats expérimentaux et numériques confirment parfaitement les déductions issues de ce modèle. Plusieurs phénomènes fondamentaux sont ainsi mis en évidence :

- présence d'un choc courbe suivi d'une compression (pour le couple $\mathrm{X} 1 / \mathrm{Be}$ ) ou d'un faisceau d'ondes de compression (pour le couple $\mathrm{T} 2 / \mathrm{Be}$ ) dans l'inerte,

- avance du front de détonation près des interfaces.

\section{REFERENCES :}

/1/ H.M. STERNBERG, D. PIACESI, Phys. Fluids 9, 1307 (1966)

/2/ G.EDEN, P.W. WRIGHT, 4ème symposium internationale sur la détonation, 573-583 (1965)

$/ 3 /$ G. EDEN, R.A. BELCHER, 9ème symposium international sur la détonation, 322-330 (1989)

/4/ T. NEAL, 6ème symposium international sur la détonation 493-502 (1976)

/5/ P. DONGUY, N. LEGRAND, 7ème symposium international sur la détonation, 386-392 (1981) 